\title{
THE MAIN CUBIOID
}

\author{
ALEXANDER BLOKH, LEX OVERSTEEGEN, ROSS PTACEK, \\ AND VLADLEN TIMORIN
}

\begin{abstract}
We discuss different analogs of the main cardioid in the parameter space of cubic polynomials, and establish relationships between them.
\end{abstract}

\section{INTRODUCTION}

For a complex polynomial $f$, let $J(f)$ be its Julia set and $K(f)$ be its filled Julia set. By classes of degree $d$ polynomials, we mean affine conjugacy classes. Denote by $[f]$ the class of a polynomial $f$.

The degree $d$ connectedness locus $\mathcal{M}_{d}$ is the set of classes of degree $d$ polynomials $f$ with connected $K(f)$ (equivalently, $[f] \in \mathcal{M}_{d}$ if all critical points of $f$ belong to $K(f))$. Thus, the set $\mathcal{M}_{2}$ is identified with the Mandelbrot set consisting of all complex parameters $c$ such that the orbit of 0 under the polynomial $z^{2}+c$ is bounded. The central part of the Mandelbrot set, the Principal Hyperbolic Domain $\mathrm{PHD}_{2}$, consists of all parameter values $c$ such that the polynomial $z^{2}+c$ is hyperbolic, and the set $K\left(z^{2}+c\right)$ is a Jordan disk (a polynomial of any degree is said to be hyperbolic if the orbits of all its critical points converge to attracting cycles). The boundary of $\mathrm{PHD}_{2}$ is called the Main Cardioid.

In degree $d$, the Principal Hyperbolic Domain $\mathrm{PHD}_{d}$ of $\mathcal{M}_{d}$ is the hyperbolic component of $\mathcal{M}_{d}$ consisting of classes $[f]$ such that $K(f)$ is a Jordan disk. Equivalently, the class $[f]$ of a degree $d$ polynomial

Date: May 24, 2013.

2010 Mathematics Subject Classification. Primary 37F45; Secondary 37F10, 37F20, 37F50.

Key words and phrases. Complex dynamics; Julia set; connectedness locus; laminations.

The first and the third named authors were partially supported by NSF grant DMS-1201450.

The second named author was partially supported by NSF grant DMS-0906316.

The fourth named author was partially supported by the Dynasty Foundation fellowship, the Simons-IUM fellowship, RFBR grants 11-01-00654-a, 12-01-33020, and AG Laboratory NRU-HSE, MESRF grant ag. 11.G34.31.0023. 
$f$ belongs to $\mathrm{PHD}_{d}$ if all critical points of $f$ are in the immediate attracting basin of the same attracting (or super-attracting) fixed point. Theorem A lists necessary conditions for $[f]$ to belong to $\overline{\mathrm{PHD}}_{d}$ (a point $b \in B$ of a topological space $B$ is called a cutpoint of $B$ if $B \backslash\{b\}$ is disconnected).

Definition 1.1 (Main Cubioid). The Main Cubioid is the set CU of classes of cubic polynomials $f$ with connected $J(f)$ such that:

(1) the polynomial $f$ has at least one non-repelling fixed point,

(2) it has no repelling periodic cutpoints of $J(f)$, and

(3) all non-repelling periodic points but perhaps one fixed point have multiplier 1.

Theorem A. Let $f$ be a polynomial, whose class belongs to $\overline{\mathrm{PHD}}_{d}$. Then $f$ has a fixed non-repelling point and no repelling periodic cutpoints of the Julia set of $f$. Moreover, all non-repelling periodic points but perhaps one fixed point have multiplier 1. Thus, $\overline{\mathrm{PHD}}_{3} \subset \mathrm{CU}$.

If $[f] \in \overline{\mathrm{PHD}}_{d}$ then $f$ cannot have two attracting periodic points as otherwise any small perturbation of $f$ will have two periodic attracting points while there exist polynomials with classes from $\mathrm{PHD}_{d}$ (and hence with only one periodic attracting point) arbitrarily close to $f$. A part of Theorem A extends this observation to non-repelling periodic points.

The name of CU suggests similarity with the Main Cardioid with one difference: the quadratic analog of the Main Cubioid is the closure of the Principal Hyperbolic Domain $\mathrm{PHD}_{2}$ rather than the boundary of it. Observe that, by definition, if $J(f)$ is disconnected, then $[f] \notin \mathrm{CU}$. Observe also that by the Fatou-Shishikura inequality [Fat20, Shi87], a cubic polynomial $f$ has at most two non-repelling cycles.

As in [BOPT13b], we now define the extended closure $\overline{\mathrm{PHD}}_{3}^{e}$ of the principal hyperbolic domain. To do so we first need to define a holomorphic motion. Let $\Lambda$ be a Riemann surface, and $Z \subset \mathbb{C}$ any (!) subset. A holomorphic motion of the set $Z$ is a map $\mu: Z \times \Lambda \rightarrow \mathbb{C}$ with the following properties:

- for every $z \in Z$, the map $\mu(z, \cdot): z \times \Lambda \rightarrow \mathbb{C}$ is holomorphic;

- for $z \neq z^{\prime}$ and every $\nu \in \Lambda$, we have $\mu(z, \nu) \neq \mu\left(z^{\prime}, \nu\right)$;

- there is a point $\nu_{0}$ such that $\mu\left(z, \nu_{0}\right)=z$ for all $z \in Z$.

A crucial result about holomorphic motions is the $\lambda$-lemma of Mañé, Sad and Sullivan [MSS83]: a holomorphic motion of a set $Z$ extends to a unique holomorphic motion of the closure $\bar{Z}$; moreover, this extension is a continuous function in two variables. Suppose that for each $\nu \in \Lambda$ a map $h_{\nu}: Z \rightarrow \mathbb{C}$ is given. A holomorphic motion $\mu: Z \times \Lambda \rightarrow \mathbb{C}$ is called 
equivariant (with respect to the family of maps $h_{\nu}$ ) if for every $\nu \in \Lambda$ and every $z \in Z$ with $h_{\nu_{0}}(z) \in Z$ we have $h_{\nu}(\mu(z, \nu))=\mu\left(h_{\nu_{0}}(z), \nu\right)$.

Let $\mathcal{F}_{\lambda}$ be the space of all cubic polynomials of the form

$$
f_{\lambda, b}(z)=\lambda z+b z^{2}+z^{3}, \quad b \in \mathbb{C} .
$$

A polynomial $f \in \mathcal{F}_{\lambda}$ is called stable with respect to 0 if its Julia set admits an equivariant holomorphic motion over some neighborhood of $f$ in $\mathcal{F}_{\lambda}$. The $\left(\lambda\right.$-) stable set $\mathcal{S}_{\lambda} \subset \mathbb{C}$ is the set of all $b \in \mathbb{C}$ such that $f_{\lambda, b}$ is stable with respect to 0 . A $\left(\lambda\right.$-) stable domain is a component $\Lambda$ of $\mathcal{S}_{\lambda}$. Clearly, for any $b_{1}, b_{2}$ in a $\lambda$-stable component $\Lambda$ there is an equivariant holomorphic motion from $J\left(f_{\lambda, b_{1}}\right)$ to $J\left(f_{\lambda, b_{2}}\right)$. A polynomial $g$ is said to be stable if $g \in[f]$ where $f \in \mathcal{F}_{\lambda}$ is stable with respect to 0 .

Definition 1.2. The set $\overline{\mathrm{PHD}}_{3}^{e}$ is the union of $\overline{\mathrm{PHD}}_{3}$ and all $\lambda$-domains of stability $\Lambda$ with $|\lambda| \leqslant 1$ such that classes of polynomials from $\operatorname{Bd}(\Lambda)$ belong to $\overline{\mathrm{PHD}}_{3}$.

We conjecture that $\overline{\mathrm{PHD}}_{3}=\mathrm{CU}=\overline{\mathrm{PHD}}_{3}^{e}$. To support this conjecture, we prove Theorem $\mathrm{B}$. Let $\mathcal{L C}$ be the set of classes of all polynomials with locally connected Julia set.

Theorem B. We have $\overline{\mathrm{PHD}}_{3}^{e} \subset \mathrm{CU}$ and $\mathcal{L C} \cap \mathrm{CU}=\mathcal{L C} \cap \overline{\mathrm{PHD}}_{3}^{e}$.

To state Theorem $\mathrm{C}$ we need to combine rational laminations [Kiw04] and laminations understood as equivalence relations on $\mathbb{S}^{1}$ (see [BL02, BCO11]). We discuss the notion of a lamination in great detail in Section 5, here we only give necessary definitions. Denote by $\mathbb{D} \subset \mathbb{C}$ the open unit disk of radius 1 centered at the origin, and by $\mathbb{S}^{1}$ the boundary of $\mathbb{D}$.

Definition 1.3 (Laminations). An equivalence relation $\sim$ on the unit circle $\mathbb{S}^{1}$ is called a lamination if either $\mathbb{S}^{1}$ is one $\sim$-class (such laminations are called degenerate), or the following holds:

(E1) the graph of $\sim$ is a closed subset in $\mathbb{S}^{1} \times \mathbb{S}^{1}$;

(E2) if $t_{1} \sim t_{2} \in \mathbb{S}^{1}$ and $t_{3} \sim t_{4} \in \mathbb{S}^{1}$, but $t_{2} \not t_{3}$, then the open straight line segments in $\mathbb{C}$ with endpoints $t_{1}, t_{2}$ and $t_{3}, t_{4}$ are disjoint; (E3) each equivalence class of $\sim$ is totally disconnected.

A lamination $\sim$ admits a canonical extension onto $\mathbb{C}$ : its classes are either convex hulls of classes of $\sim$, or points which do not belong to such convex hulls. By Moore's Theorem the space $\mathbb{C} / \sim$ is homeomorphic to $\mathbb{C}$. The quotient map $p_{\sim}: \mathbb{S}^{1} \rightarrow \mathbb{S}^{1} / \sim$ extends to the plane with the only non-trivial point-preimages (fibers) being the convex hulls of $\sim$-classes. From now on we will always consider such extensions of the quotient map. We write $\sigma_{d}: \mathbb{S}^{1} \rightarrow \mathbb{S}^{1}$ for the map $z \mapsto z^{d}$. 
Definition 1.4 (Laminations and dynamics). A lamination $\sim$ is called $\left(\sigma_{d^{-}}\right)$invariant if:

(D1) $\sim$ is forward invariant: for a $\sim$-class $\mathbf{g}$, the set $\sigma_{d}(\mathbf{g})$ is a $\sim$-class; (D2) for any $\sim$-class $\mathbf{g}$, the map $\sigma_{d}: \mathbf{g} \rightarrow \sigma_{d}(\mathbf{g})$ extends to $\mathbb{S}^{1}$ as an orientation preserving covering map such that $\mathbf{g}$ is the full preimage of $\sigma_{d}(\mathbf{g})$ under this covering map.

For a $\sigma_{d}$-invariant lamination $\sim$ consider the topological Julia set $\mathbb{S}^{1} / \sim=J_{\sim}$ and the topological polynomial $f_{\sim}: J_{\sim} \rightarrow J_{\sim}$ induced by $\sigma_{d}$. One can extend $f_{\sim}$ to a branched-covering map $f_{\sim}: \mathbb{C} \rightarrow \mathbb{C}$ of degree $d$ called a topological polynomial too. The map $p_{\sim}$ semiconjugates $\sigma_{d}$ with $f_{\sim}$, at least on the unit circle and all leaves of $\sim$. Unlike complex polynomials, topological polynomials can have periodic critical points in their topological Julia sets. The complement $K_{\sim}$ of the unique unbounded component $U_{\infty}\left(J_{\sim}\right)$ of $\mathbb{C} \backslash J_{\sim}$ is called the filled topological Julia set. For $a, b \in \mathbb{S}^{1}$, let $\overline{a b}$ be the chord with endpoints $a$ and $b$. If $A \subset \mathbb{S}^{1}$ is closed, boundary chords of the convex hull $\mathrm{CH}(A)$ of $A$ are called edges of $\mathrm{CH}(A)$.

Definition 1.5 (Leaves and gaps). If $A$ is a $\sim$-class, call an edge $\overline{a b}$ of $\operatorname{Bd}(\mathrm{CH}(A))$ a leaf. All points of $\mathbb{S}^{1}$ are also called (degenerate) leaves. The family $\mathcal{L}_{\sim}$ of all leaves of $\sim$ is called the geometric lamination (geo-lamination) generated by $\sim$. Let $\mathcal{L}_{\sim}^{+}$be the union of all leaves of $\mathcal{L}_{\sim}$. The closure of a non-empty component of $\mathbb{D} \backslash \mathcal{L}_{\sim}^{+}$is called a gap of $\sim$. Leaves and gaps of $\mathcal{L}_{\sim}$ are called $\mathcal{L}_{\sim}$-sets; a leaf which is not an edge of a finite gap is called independent. If $G$ is a gap or leaf, we call the set $G^{\prime}=\mathbb{S}^{1} \cap G$ the basis of $G$.

Extend $\sigma_{d}$ (keeping the notation) linearly over all individual chords in $\overline{\mathbb{D}}$ (e.g., over leaves of $\mathcal{L}_{\sim}$ ); even though the extended $\sigma_{d}$ is not welldefined on the entire disk, it is well-defined on $\mathcal{L}_{\sim}^{+}$. A gap or leaf $U$ is said to be (pre)periodic if $\sigma_{d}^{m+k}\left(U^{\prime}\right)=\sigma_{d}^{m}\left(U^{\prime}\right)$ for some $m \geqslant 0, k>0$. If $m$ above can be chosen to be 0 , then $U$ is called periodic; the minimal number $k$ above is called the period of $U$. If $U$ is (pre)periodic but not periodic then it is called preperiodic. A Fatou gap is a gap with infinite basis. By [Kiw02] a Fatou gap $G$ is (pre)periodic under $\sigma_{d}$.

Definition 1.6 (Rotational sets and numbers). If $\mathbf{g}$ is a periodic nondegenerate finite $\sim$-class of period $n$, the map $\sigma_{d}^{\circ n} \mid \mathrm{g}$ is conjugate (by a conjugacy that preserves the cyclic order) to a rigid rotation $R_{\rho}$ by a rational angle $\rho$ on a finite $R_{\rho}$-invariant subset of $\mathbb{S}^{1}$. The number $\rho$ is then called the rotation number of $\mathbf{g}$. A periodic Fatou gap $G$ of period $n$ such that $\left.f_{\sim}^{\circ n}\right|_{\operatorname{Bd}\left(p_{\sim}(G)\right)}$ is conjugate to an irrational rotation by an angle $\rho$, is called a Siegel gap while $\rho$ is called the rotation number of 
$G$. Otherwise $\left.f_{\sim}^{\circ n}\right|_{\operatorname{Bd}\left(p_{\sim}(G)\right)}$ is conjugate to a map $\sigma_{k}$ with some $k>1$ and $G$ is called a Fatou gap of degree $k$. Siegel gaps and finite $\sim$-classes with non-zero rotation number are called rotational sets.

In Section 5 we combine ideas from Kiw04 and [BCO11] and define the geo-lamination $\mathcal{L}_{f}$ associated to $f$. First we associate to $f$ a lamination $\sim_{f}$ such that the monotone map from $J(f)$ onto $J_{\sim_{f}}$ is the finest monotone map of $J(f)$ onto a locally connected continuum (a map is monotone if point-preimages - fibers - are continua). This is possible by [BCO11 (see Theorems 4.1 and 4.4 in Section 44). To specify $\mathcal{L}_{\sim_{f}}$ we need the following definition.

Definition 1.7. A curve $\Gamma$ in the dynamic plane of $f$ consisting of dynamic (periodic of period $k$ ) external rays $R_{f}\left(\theta_{1}\right), R_{f}\left(\theta_{2}\right)$ and their common landing point $x$ is called a (periodic of period $k$ ) cut while $x$ is called the vertex of $\Gamma$.

Finally, we add to $\mathcal{L}_{\sim_{f}}$ leaves and finite gaps corresponding to periodic cuts of the fibers associated to infinite classes of $\sim_{f}$. It was shown in [BCO11] that a fiber contains at most finitely many vertices of periodic cuts. We also add the corresponding pullbacks of the added leaves and gaps. We make a distinction between different kinds of gaps of $\mathcal{L}_{f}$ depending on what they correspond to in terms of $f$. Thus, to each polynomial we associate a geo-lamination $\mathcal{L}_{f}$ equipped with an additional structure: for each gap or leaf of $\mathcal{L}_{f}$, we know if it is contained in the convex hull of a $\sim_{f}$-class or not.

Definition 1.8. A laminational pair is a pair $\{\sim, \mathcal{L}\}$ where $\mathcal{L} \supset \mathcal{L} \sim$ is a geo-lamination obtained by adding to $\mathcal{L}_{\sim}$ finitely many finite periodic gaps or leaves inside convex hulls of infinite $\sim$-classes as well as all their preimages so that $\mathcal{L}$ is a geo-lamination.

Now we can define the Combinatorial Main Cubioid.

Definition 1.9. A cubic lamination $\sim\left(\right.$ and its geo-lamination $\left.\mathcal{L}_{\sim}\right)$ is cubioidal if each periodic non-degenerate leaf of $\sim$ has an attached to it Fatou gap whose basis is not one $\sim$-class, and $\sim$ has at most one rotational set. A laminational pair $\{\sim, \mathcal{L}\}$ is cubioidal if $\sim$ is cubioidal and $\mathcal{L}_{\sim}=\mathcal{L}$. The Combinatorial Main Cubioid $\mathrm{CU}^{c}$ is the set of all cubioidal geo-laminations (they are called CU-laminations).

There are two extreme cases for $\left\{\sim_{f}, \mathcal{L}_{f}\right\}$. First, $\sim_{f}$ may identify no two points. Then $J(f)$ is a Jordan curve, $\mathcal{L}_{f}$ has no leaves, and $[f] \in \mathrm{CU}$. We call such laminational pair empty. Second, $\sim_{f}$ may identify all points of $\mathbb{S}^{1}$ while $\mathcal{L}_{f}$ contains no leaves. By our Lemma 5.1] then 
again $[f] \in \mathrm{CU}$. We call such laminational pair degenerate. The degenerate and the empty laminational pairs share the same geo-lamination, are cubioidal, and correspond to polynomials $f$ with $[f] \in \mathrm{CU}$, yet correspond to two very different types of dynamics. In all other cases $\mathcal{L}_{f}$ includes some non-degenerate leaves.

Theorem C. If $[f] \in \mathrm{CU}$ then $\left(\sim_{f}, \mathcal{L}_{f}\right)$ is a cubioidal laminational pair.

Notation: we write $\bar{A}$ for the closure of a subset $A$ of a topological space and $\operatorname{Bd}(A)$ for the boundary of $A$; the $n$-th iterate of a map $f$ is denoted by $f^{\circ n}$. For $A \subset \mathbb{C}$ let $\mathrm{CH}(A)$ be the convex hull of $A$ in $\mathbb{C}$. If it does not cause ambiguity, we will often speak of cutpoints meaning cutpoints of the appropriate Julia sets. We will consistently identify angles, i.e. elements of $\mathbb{R} / \mathbb{Z}$, with points of the unit circle $\mathbb{S}^{1} \subset \mathbb{C}$.

\section{Proof of Theorem A}

We first recall some terminology and notation.

2.1. Dynamic rays. Let $f(z)=z^{d}+a_{d-1} z^{d-1}+\cdots+a_{0}$ be a monic degree $d$ polynomial. The Green function $G_{f}$ is defined by the formula

$$
G_{f}(z)=\lim _{n \rightarrow \infty} \frac{\log _{+}\left|f^{\circ n}(z)\right|}{d^{n}},
$$

where $\log _{+} r$ equals $\log r$ if $r>0$ and 0 otherwise. This function is harmonic on the complement of the filled Julia set $K(f)$ of $f$ and is equal to 0 on $K(f)$. Define dynamic rays as unbounded trajectories of the gradient flow for $G_{f}$. Let $V(f)$ be the union of all dynamic rays of $f$. Then $V(f)$ is a forward-invariant open set, and there is a conformal isomorphism $\phi_{f}$ between $V(f)$ and some open subset of the set $\{|z|>1\}$ with the following properties:

$$
\phi_{f}(f(z))=\phi_{f}(z)^{d}, \quad G_{f}(z)=\log \left|\phi_{f}(z)\right| .
$$

These properties define the map $\phi_{f}$ almost uniquely: the only way to change the map $\phi_{f}$ without violating the two properties is to postcompose it with multiplication by a $(d-1)$-st root of unity.

The map $\phi_{f}$ is called a Bötcher coordinate. It is used to parameterize dynamic rays of $f$. Every dynamic ray is the preimage of a straight radial ray $\left\{r e^{2 \pi i \theta} \mid r>r_{0}\right\}, r_{0} \geqslant 1$, under the map $\phi_{f}$ (if $K(f)$ is disconnected then $r_{0}>1$ if the dynamic ray contains a pre-critical point in its closure). We will write $R_{f}(\theta)$ for this ray, and call it the dynamic ray of argument $\theta$. Arguments of dynamic rays are angles, i.e. elements of the group $\mathbb{R} / \mathbb{Z}$. If $f$ is a degree $d$ polynomial, not 
necessarily monic, then we can make it monic by a complex linear change of variables. Thus, it still makes sense to talk about dynamic rays of $f$.

However, arguments of dynamic rays are not well-defined, since they depend on the choice of a Böttcher coordinate. Hence every time we consider rays in dynamic planes of different polynomials, we must resolve the issue of choosing the arguments consistently. For example, if a sequence $f_{n}$ of degree $d$ polynomials converges to a degree $d$ polynomial $f$, then we can choose any Böttcher coordinate for $f$, and then, for $f_{n}$ sufficiently close to $f$, choose the Böttcher coordinate for $f_{n}$ that is close to the chosen Böttcher coordinate for $f$.

Suppose that the Julia set $J(f)$ is connected. Consider a periodic repelling cutpoint $\alpha$ of $f$, and let $r$ be its minimal period. Then there are finitely many dynamic rays landing at $\alpha$; we will assume that the choice of their arguments is fixed, and denote the set of the arguments by $\operatorname{Ar}_{f}(\alpha)$. Every wedge between consecutive rays landing at $\alpha$ contains exactly one component of $J(f) \backslash\{\alpha\}$. The dynamic rays landing at $\alpha$ may form one or more orbits under the map $f^{\circ}$. Choose one of the orbits, and let $\theta_{0}, \ldots, \theta_{q-1}$ denote the arguments of all rays in this orbit labeled in the counterclockwise order. Suppose that $d^{r} \cdot \theta_{i}=\theta_{i+p}(\bmod q)$, where $d$ is the degree of the polynomial $f$. In this case, we say that $\alpha$ has combinatorial rotation number $p / q$; observe that $p, q$ are coprime except for the case when $p / q=0$ and all rays landing at $\alpha$ are invariant. Every repelling fixed point has a well-defined combinatorial rotation number (thus, $p / q$ above does not depend on the choice of $\theta_{0}$ ).

2.2. Polynomials in $\overline{\mathrm{PHD}}_{d}$. We now recall Lemma B.1 from [GM93. that goes back to Douady and Hubbard [DH8485].

Lemma 2.1. Let $f$ be a polynomial, and $z$ be a repelling periodic point of $f$. If the ray $R_{f}(\theta)$ lands at $z$, then, for every polynomial $g$ sufficiently close to $f$, the ray $R_{g}(\theta)$ lands at a repelling periodic point $w$ close to z. Moreover, $w$ depends holomorphically on $g$.

Choose a polynomial $f$ with $[f] \in \overline{\mathrm{PHD}}_{d}$. For brevity, by a cutpoint we mean a cutpoint of $J(f)$. We want to prove the following statements:

(1) the map $f$ has no repelling periodic cutpoints, and

(2) the map $f$ has at most one non-repelling periodic point of multiplier different from 1.

Statement (1) follows from Lemma 2.1. Indeed, suppose that $\alpha$ is a repelling periodic cutpoint of $f$. Let $\theta_{0}, \ldots, \theta_{r-1}$ be the arguments of dynamic rays landing at $\alpha$. Since $\alpha$ is a cutpoint, then $r>1$. Lemma 2.1 says that, for $g$ sufficiently close to $f$, the dynamic rays 
with arguments $\theta_{0}, \ldots, \theta_{r-1}$ in the dynamic plane of $g$ land at the same periodic point that is obtained from $\alpha$ by analytic continuation. We get a contradiction if we choose $g$ such that $[g] \in \mathrm{PHD}_{d}$.

The second statement is a consequence of the Yoccoz inequality, see e.g. Hub93. It follows from the Yoccoz inequality that, for any sequence of polynomials $f_{n}$ with repelling fixed points $\alpha_{n} \rightarrow \alpha$, the fact that $f_{n}^{\prime}\left(\alpha_{n}\right) \rightarrow e^{2 \pi i \rho}$ implies that the combinatorial rotation numbers of $f_{n}$ at $\alpha_{n}$ converge to $\rho$. We can now prove Lemma 2.2.

Lemma 2.2. Consider a polynomial $f$, whose class belongs to $\overline{\mathrm{PHD}}_{d}$, and a sequence of polynomials $f_{n}$ converging to $f$, whose classes belong to $\mathrm{PHD}_{d}$. If $f$ has a non-repelling fixed point $\alpha$, whose multiplier is different from 1, then $\alpha$ is the limit of the attracting fixed points of $f_{n}$.

Proof. Let $\alpha$ be neutral and $\alpha_{n}$ be a fixed point of $f_{n}$ such that $\alpha_{n} \rightarrow \alpha$ as $n \rightarrow \infty$. If $\alpha_{n}$ are attracting for arbitrarily large $n$, then we are done. Otherwise assume that for all large $n$, the points $\alpha_{n}$ are repelling fixed points. Then their combinatorial rotation numbers equal 0 . By the assumptions, $f^{\prime}(\alpha)=e^{2 \pi i \rho}$, where $\rho \not \equiv 0(\bmod 2 \pi)$. On the other hand, by the Yoccoz inequality, the combinatorial rotation numbers of $f_{n}$ at $\alpha_{n}$ (which are all equal to 0 ) must converge to $\rho$, a contradiction.

We can now complete the proof of Theorem A.

Proof of Theorem A. Observe that if $[f] \in \overline{\mathrm{PHD}}_{d}$ then one of the nonrepelling cycles of $f$ must be a fixed point (indeed, as we approximate $f$ with polynomials $g$, whose classes belong to $\mathrm{PHD}_{d}$, the attracting fixed points of $g$ converge to a non-repelling fixed point of $f$ ). Let $[f] \in \overline{\mathrm{PHD}}_{d}$. By way of contradiction, suppose that $\alpha$ and $\beta$ are two non-repelling periodic points, whose multipliers are different from one. Replacing $f$ with a suitable iterate, we may assume that $\alpha$ and $\beta$ are fixed. At least one of the two points, say, $\alpha$, is not the limit of the attracting fixed points of polynomials $g$ with $[g] \in \mathrm{PHD}_{d}$ approximating the polynomial $f$. But this is a contradiction with Lemma 2.2 .

\section{Proof of the first part of Theorem B}

By definition, all polynomials from $\overline{\mathrm{PHD}}_{3}^{e}$ have a fixed non-repelling point $\alpha$. Mapping $\alpha$ to 0 by an affine transformation, rotating and rescaling if necessary, we reduce polynomials from $\overline{\mathrm{PHD}}_{3}^{e}$ to the form

$$
f_{\lambda, b}(z)=\lambda z+b z^{2}+z^{3},|\lambda| \leqslant 1 .
$$

Let $\mathcal{F}$ denote the space of all cubic polynomials of the form $f_{\lambda, b}$ and $\mathcal{F}_{n r}$ denote the subspace of $\mathcal{F}$ consisting of polynomials $f_{\lambda, b}$ with $|\lambda| \leqslant 1$. 
Fix $\lambda$ with $|\lambda| \leqslant 1$. Let $\mathfrak{g}_{\lambda, b}$ be the Green function for $K\left(f_{\lambda, b}\right)$. Let $V_{\lambda, b}$ be the union of all unbounded trajectories of the gradient flow generated by $\mathfrak{g}_{\lambda, b}$. The Böttcher coordinate is an analytic map $\phi_{\lambda, b}: V_{\lambda, b} \rightarrow \mathbb{C}$ with $\phi_{\lambda, b} \circ f_{\lambda, b}=\phi_{\lambda, b}^{3}$ and $\phi_{\lambda, b}(z)=z+o(z)$ as $z \rightarrow \infty$.

Theorem 3.1 ([BuHe01], Proposition 2). Let $\mathcal{V}_{\lambda}$ be the union of $\{b\} \times$ $V_{\lambda, b}$ over all $b \in \mathbb{C}$. This set is open in $\mathbb{C}^{2}$. The map $\Phi_{\lambda}: \mathcal{V}_{\lambda} \rightarrow \mathbb{C}^{2}$ given by the formula $\Phi_{\lambda}(b, z)=\left(b, \phi_{\lambda, b}(z)\right)$ is an analytic embedding of $\mathcal{V}$ into $\mathbb{C}^{2}$.

We will write $R_{\lambda, b}(\theta)$ for the dynamic ray $R_{f_{\lambda, b}}(\theta)$.

3.1. Polynomials with parabolic points and their petals. Let $g$ be a polynomial of arbitrary degree such that 0 is a fixed parabolic point of $g$ of multiplier 1. Suppose that $g(z)=z+a z^{q+1}+o\left(z^{q+1}\right)$, where $q$ is a positive integer. Recall from [Mil06] that an attracting vector for $g$ is defined as a vector (=complex number) $v$ such that $a v^{q}$ is a negative real number, i.e. $v$ and $a v^{q+1}$ have opposite directions. Clearly, there are $q$ straight rays consisting of attracting vectors that divide the plane of complex numbers into $q$ repelling sectors.

Consider a repelling sector $S$. Note that the set $S^{-q}=\left\{z \in \mathbb{C} \mid z^{-q} \in\right.$ $S\}$ is the complement of the ray $\{-t a \mid t>0\}$ in $\mathbb{C}$. Let $U$ be a sufficiently small disk around 0 . We will write $F$ for the composition of the function $w \mapsto w^{-1 / q}$ mapping $(S \cap U)^{-q}$ onto $S \cap U$, the function $g$ mapping $S \cap U$ onto $g(S \cap U)$, and the function $z \mapsto z^{-q}$ mapping $g(S \cap U)$ to $\mathbb{C}$. We have $F(w)=w-q a+\alpha(w)$, where $\alpha(w)$ denotes a power series in $w^{-1 / q}$ that converges in a neighborhood of infinity, and whose free term is zero (note that this function is single valued and holomorphic on $\left.(S \cap U)^{-q}\right)$. It follows that there exists a positive real number $r$ with the property $|\alpha(w)|<\frac{|a|}{2}$ whenever $|w|>r|a|$. Consider the half-plane $\Pi$ given by the inequality $\operatorname{Re}(w / a)>r$. Since this inequality implies that $|w|>r|a|$, we have $F(\Pi) \supset \Pi$, and also that the shortest distance from a point on the boundary of $\Pi$ to a point on the boundary of $F(\Pi)$ is at least $\left(q-\frac{1}{2}\right)|a|$. The preimage $R P$ of the half-plane $\Pi$ under the map $z \mapsto z^{-q}$ from $S$ to $S^{-q}$ is called a repelling petal of $g$.

Every repelling sector includes a repelling petal; thus, our polynomial $g(z)=z+a z^{q+1}+o\left(z^{q+1}\right)$ has $q$ repelling petals. A repelling petal $R P$ of $g$ is such that $g(R P) \supset R P$. Let us discuss the dependence of the repelling petals on parameters. Suppose that a polynomial $\tilde{g}(z)=$ $z+\tilde{a} z^{q+1}+\ldots$ is very close to the polynomial $g(z)$, in particular, the coefficients $a$ and $\tilde{a}$ are very close to each other. We will assume that $a$ is nonzero, and that $\tilde{a} / a$ is close to 1 . Consider repelling sectors $S$ 
and $\tilde{S}$ of $g$ and $\tilde{g}$, respectively. Since $a$ and $\tilde{a}$ are close, we can choose the repelling sectors $S$ and $\tilde{S}$ to be close.

The half-planes $\Pi$ and $\tilde{\Pi}$ associated with polynomials $g$ and $\tilde{g}$ in the same way as above (we may choose the same sufficiently large $r$ for both $g$ and $\tilde{g}$ ) are also close in the Hausdorff metric associated with the spherical metric (although this is not true for the Euclidean metric). The preimages of $\Pi$ and $\tilde{\Pi}$ under the map $z \mapsto z^{-q}$ acting on $S^{-q}$ and $\tilde{S}^{-q}$, respectively, are also close. Thus we obtain the following lemma (cf. the proof of Lemma 5 in [BuHe01]).

Lemma 3.2. Let $g_{t}(z)=z+a_{t} z^{q+1}+o\left(z^{q+1}\right)$ be a continuous family of polynomials, in which $a_{t}$ never vanishes. Then all $q$ repelling petals of $g_{t}$ can be chosen to vary continuously with respect to the parameter.

3.2. Stability of rays and their perturbations. Throughout this subsection, we fix $\lambda$ that is a root of unity, i.e. $\lambda=\exp (2 \pi i p / q)$ for some relatively prime $p$ and $q$. Since $\lambda$ is fixed, we will skip $\lambda$ from the notation $f_{b}, R_{b}(\theta)$ etc. We discuss conditions that guarantee that a dynamical ray $R_{b}(\theta)$ landing at 0 is stable, i.e., for $b^{\prime}$ sufficiently close to $b$, the ray $R_{b^{\prime}}(\theta)$ also lands at 0 .

Proposition 3.3. We have $f_{b}^{\circ q}(z)=z+T_{p / q}(b) z^{q+1}+o\left(z^{q+1}\right)$, where $T_{p / q}(b)$ is a non-zero polynomial in $b$.

Proof. By the Petal Theorem [Bea00, Theorem 6.5.10], we have

$$
f_{b}^{\circ q}(z)=z+T_{p / q}(b) z^{q+1}+o\left(z^{q+1}\right) .
$$

It remains to prove that the polynomial $T_{p / q}(b)$ cannot be identically equal to zero. For any $b$ such that $T_{p / q}(b)=0$, the polynomial $f_{b}$ has at least two cycles of attracting petals at 0. Each of the associated cycles of Fatou domains must contain a critical point of $f_{b}$. Thus both critical orbits of $f_{b}$ converge to 0 . However, for large $b$, one of the critical points escapes. Therefore, for such $b$, we have $T_{p / q}(b) \neq 0$.

Proposition 3.4. Suppose that a dynamic ray $R_{b_{*}}(\theta)$ with periodic $\theta$ lands at 0 , and $T_{p / q}\left(b_{*}\right) \neq 0$. Then, for all $b$ sufficiently close to $b_{*}$, the ray $R_{b}(\theta)$ lands at 0 .

Proof. By Lemma 10.1 of [Mil06], the ray $R_{b_{*}}(\theta)$ must be tangent to some repelling vector of $f_{b_{*}}^{\circ q}$ at 0 . Let $R P_{b_{*}}$ be the corresponding repelling petal of $f_{b_{*}}^{\circ q}$. The period of $\theta$ is equal to $q$ by Theorem 18.13 of [Mil06]. There are two points $z_{*}$ and $f_{b_{*}}^{\circ q}\left(z_{*}\right)$ in $R_{b_{*}}(\theta)$ that lie in the interior of $R P_{b_{*}}$. By Lemma 3.2 for all $b$ sufficiently close to $b_{*}$, we can define a repelling petal $R P_{b}$ of $f_{b}^{\circ q}$ that is close to $R P_{b_{*}}$ in the Hausdorff metric. 
Let $L_{*}$ denote the subray of the ray $R_{b_{*}}(\theta)$ from $z_{*}$ to infinity. By Theorem [3.1, for every $\varepsilon>0$, we can choose a neighborhood $U$ of $b_{*}$ such that, for all $b \in U$, the corresponding piece $L$ of $R_{b}$ is $\varepsilon$-close to $L_{*}$ in the Hausdorff metric. The number $\varepsilon$ can be chosen so that this implies that $L$ enters the corresponding petal $R P_{b}$. Dynamics inside $R P_{b}$ implies that $R_{b}(\theta)$ lands at 0 .

3.3. The proof of inclusion $\overline{\mathrm{PHD}}_{3}^{e} \subset \mathrm{CU}$. By Theorem A, we have $\mathrm{PHD}_{3} \subset \mathrm{CU}$. Consider a two-dimensional domain $\mathcal{U} \subset \mathcal{F}_{\lambda}$ consisting of stable polynomials such that for all $f \in \operatorname{Bd}(\mathcal{U})$, we have $[f] \in \overline{\mathrm{PHD}}_{3}$. We need to prove that classes of all polynomials in $\mathcal{U}$ belong to CU. Suppose that $f_{*}=f_{\lambda, b_{*}} \in \mathcal{U}$, and show that then $f_{*}$ has properties (1)-(3) from Definition 1.1.

Property (1). Clearly, $f_{*}$ has a fixed non-repelling point 0 , thus property (1) is fulfilled.

Property (2). Let us prove that $f_{*}$ has no repelling cutpoints. Assume that $f_{*}$ has a repelling periodic cutpoint $z_{b_{*}}$. The set $\operatorname{Ar}_{f_{*}}\left(z_{b_{*}}\right)$ of arguments of external rays of $f_{*}$ landing at $z_{b_{*}}$ consists of at least two angles. Since all maps in $\mathcal{U}$ are quasi-symmetrically conjugate, it is easy to see (e.g., by Lemma 3.5 [BOPT13b]) that all maps $f_{\lambda, b} \in \mathcal{U}$ have repelling periodic cutpoints $z_{b}$ corresponding to $z_{b_{*}}$. By Lemma 2.1 $\operatorname{Ar}_{f_{\lambda, b}}\left(z_{b}\right)=\operatorname{Ar}_{f_{*}}\left(z_{b_{*}}\right)$. Suppose that $\{\alpha, \beta\} \subset \operatorname{Ar}_{f_{*}}\left(z_{b_{*}}\right)$.

Let $\Lambda$ be the set of all parameter values $b$ with $f_{\lambda, b} \in \mathcal{U}$, and choose a sequence $b_{n} \rightarrow b^{\prime} \in \operatorname{Bd}(\Lambda)$. We may assume that $z_{b_{n}} \rightarrow z_{b^{\prime}}$, where $z_{b^{\prime}}$ is a non-attracting periodic point of $f_{\lambda, b^{\prime}}$. If both rays $R_{b^{\prime}}(\alpha), R_{b^{\prime}}(\beta)$ land at repelling periodic points, then these landing points must coincide as otherwise by Lemma 2.1 we get a contradiction with the fact that $R_{b_{n}}(\alpha), R_{b_{n}}(\beta)$ land at $z_{b_{n}}$ and $z_{b_{n}} \rightarrow z_{b^{\prime}}$. However, by Theorem A, the map $f_{\lambda, b^{\prime}}$ does not have repelling periodic cutpoints. Hence one of the rays $R_{b^{\prime}}(\alpha), R_{b^{\prime}}(\beta)$ lands at a parabolic periodic point. Clearly, for at most finitely many parameter values $b^{\prime} \in \operatorname{Bd}(\Lambda)$ the rays $R_{b^{\prime}}(\alpha)$ or $R_{b^{\prime}}(\beta)$ land at a parabolic point distinct from 0 . Assume that for infinitely many $b^{\prime} \in \operatorname{Bd}(\Lambda)$ the rays $R_{b^{\prime}}(\alpha)$ land at 0 which is a parabolic fixed point: $\lambda=\exp (2 \pi i p / q)$ for some relatively prime $p$ and $q$.

Let us show that in the above case $T_{p / q}\left(b^{\prime}\right)=0$. Indeed, arbitrarily close to $b^{\prime}$, there are parameter values $b$, for which $R_{b}(\alpha)$ does not land at 0. It follows from Proposition 3.4 that $T_{p / q}\left(b^{\prime}\right)=0$. However, the polynomial $T_{p / q}$ has only finitely many roots, a contradiction. 
Property (3). Suppose that $f_{*}$ has a non-repelling $n$-periodic point $z_{b_{*}} \neq 0$ with multiplier not equal to 1 . Since $f_{*}$ is stable, the corresponding periodic point $z_{b} \neq 0$ of $f_{b}, b \in \Lambda$, is $n$-periodic and nonrepelling. If $b \rightarrow b^{\prime} \in \operatorname{Bd}(\Lambda)$, then $z_{b} \rightarrow z_{b^{\prime}}$ where $z_{b^{\prime}}$ is a non-repelling $f_{b^{\prime}}^{o n}$-fixed point. Consider two cases. First, suppose that $z_{b^{\prime}} \neq 0$. Then by Theorem $\mathrm{A}$ the multiplier at $z_{b^{\prime}}$ is 1 . There are only finitely many values of $b^{\prime}$, for which this can happen. Second, suppose that $z_{b^{\prime}}=0$. We have $f_{b}^{o n}(z)-z=z\left(z-z_{b}\right) Q_{b}(z)$ for some polynomial $Q_{b}$, whose coefficients are algebraic functions of $b$ that have no poles in $\mathbb{C}$. We obtain in the limit as $b \rightarrow b^{\prime}$ that $f_{b^{\prime}}^{\text {on }}(z)-z=z^{2} Q_{b^{\prime}}(z)$, hence 0 is a parabolic fixed point of $f_{b^{\prime}}$. We may assume that the multiplier at 0 is $e^{2 \pi i p / q}$. Let us show that then 0 is a degenerate parabolic point (i.e., that $T\left(b^{\prime}\right)=0$ where $T=T_{p / q}$ is the polynomial introduced in Proposition 3.3).

Indeed, $n=m q$ is a multiple of $q$, and as in Proposition 3.3 by the Petal Theorem $f_{b}^{\circ q}(z)=z+T(b) z^{q+1}+o\left(z^{q+1}\right)$. It is easy to see by induction that then for any $k$ we have $f_{b}^{\circ k q}(z)=z+k T(b) z^{q+1}+o\left(z^{q+1}\right)$. On the other hand, as above $f_{b}^{\circ m q}(z)-z=z^{q+1}\left(z-z_{b}\right) R_{b}(z)$ where $R_{b}(z)$ is a polynomial of $z$ whose coefficients are algebraic functions of $b$ that have no poles in $\mathbb{C}$. Hence in the limit we have $f_{b^{\prime}}^{o m q}(z)-z=$ $z^{q+2} R_{b^{\prime}}(z)$. It follows that $m T\left(b^{\prime}\right) z^{q+1}+o\left(z^{q+1}\right)=z^{q+2} R_{b^{\prime}}(z)$ and hence $m T\left(b^{\prime}\right)+o(1)=z R_{b^{\prime}}(z)$, which implies that $T\left(b^{\prime}\right)=0$ as desired. Clearly, there are finitely many such values of $b^{\prime}$. Thus, we showed that overall there are only finitely many values of $b^{\prime}$ to which $b$ may converge, a contradiction with $\operatorname{Bd}(\Lambda)$ being infinite.

\section{LAMINATIONS ASSOCIATED TO POLYNOMIALS}

If $X \subset \mathbb{C}$ is a continuum, let $U_{\infty}(X)$ be the unbounded component of $\mathbb{C} \backslash X$. If $X=\operatorname{Bd}\left(U_{\infty}(X)\right)$, we call $X$ unshielded. A continuous map $\varphi: Y \rightarrow Z$ is monotone if all fibers are continua. Let $A$ be a continuum. A monotone onto map $\varphi: A \rightarrow Y_{\varphi, A}$ with locally connected $Y_{\varphi, A}$ is called a finest (monotone) map if for any monotone map $\psi$ : $A \rightarrow L$ onto a locally connected continuum $L$ there is a map $h$ : $Y_{\varphi, A} \rightarrow L$ with $\psi=h \circ \varphi$ (then $h$ is monotone because for $x \in L$, we have $\left.h^{-1}(x)=\varphi\left(\psi^{-1}(x)\right)\right)$. If $\varphi: A \rightarrow B, \varphi^{\prime}: A \rightarrow B^{\prime}$ are two finest maps, then the map associating points $\varphi(x) \in B$ and $\varphi^{\prime}(x) \in B^{\prime}$ for every $x \in A$ is a homeomorphism between $B$ and $B^{\prime}$. Hence all sets $Y_{\varphi, A}$ are homeomorphic, all finest maps $\varphi$ are the same up to a homeomorphism, and we can talk of the finest model $Y_{A}=Y$ of $A$ and the finest map $\varphi_{A}=\varphi$ of $A$ onto $Y$. Recall that given a lamination $\sim$ the corresponding quotient map from $\mathbb{S}^{1}$ to $\mathbb{S}^{1} / \sim$ is denoted by $p_{\sim}$. 
Theorem 4.1 (Theorem 1 [BCO11]). Let $Q$ be an unshielded continuum. Then there exist the finest map $\varphi$ and the finest model $Y$ of $Q$ given by a lamination $\sim_{Q}$ on $\mathbb{S}^{1}$ so that $Y=\mathbb{S}^{1} / \sim_{Q}$; moreover, $\varphi$ can be extended to a map $\varphi: \mathbb{C} \rightarrow \mathbb{C}$ which collapses only those complementary domains to $Q$ whose boundaries are collapsed by $\varphi$, and is a homeomorphism elsewhere in $\mathbb{C} \backslash Q$. For $y \in Y$ the fiber $\varphi^{-1}(y)$ coincides with the topological hull of the union of impressions of all external to $Q$ rays with arguments from the set $p_{\sim_{Q}}^{-1}(y)$. $\mathbb{C}$.

By a finest map we mean any extension of the finest map of $Q$ over

Definition 4.2 (Critical leaves and gaps). A leaf of a lamination $\sim$ is called critical if its endpoints have the same image. A gap $G$ is said to be critical if $\left.\sigma_{d}\right|_{G^{\prime}}$ is at least $k$-to-1 for some $k>1$.

Lemma 4.3 is well known; we state it here without a proof.

Lemma 4.3. An edge of a periodic gap is either (pre)critical or (pre)periodic.

Laminations give a full and exact description of the dynamics of complex polynomials with locally connected Julia set [Thu85]. In general, weaker results can be proven [Kiw04, BCO11. Namely, in [BCO11] Theorem 4.1 is applied to polynomials with connected Julia set which yields Theorem 4.4 (a similar earlier result is due to Kiwi [Kiw04).

Theorem 4.4 ([BCO11], Theorem 2). Let $f$ be a complex polynomial with connected Julia set and finest lamination $\sim_{f}=\sim_{J(f)}$. Then there exists a topological polynomial $f_{\sim_{f}}: \mathbb{C} \rightarrow \mathbb{C}$ and a finest map $\varphi_{f}: \mathbb{C} \rightarrow$ $\mathbb{C}$ which semiconjugates $f$ and $f_{\sim_{f}}$. If $x \in J_{\sim_{f}}$ corresponds to a finite periodic $\sim_{f}$-class $p_{\sim_{f}}^{-1}(x)$ then the fiber $\varphi_{f}^{-1}(x)$ is a point. No periodic Fatou domain of $f$ of degree greater than 1 is collapsed by $\varphi_{f}$.

We need the following definition.

Definition 4.5. Call gaps finite or infinite if their bases are finite or infinite; infinite $\sim$-classes have infinite gaps as their convex hulls (such gaps will be called infinite gap-classes). By [BL02] all such gaps are (pre)periodic, and periodic infinite gap-classes are Fatou gaps of degree greater than 1. Call the corresponding fibers $C S$-fiber. Thus, if $x \in J_{\sim_{f}}$ corresponds to an infinite gap-class $p_{\sim_{f}}^{-1}(x)$ then the $x$-fiber $\varphi_{f}^{-1}(x)$ is said to be a $C S$-fiber.

The following lemma explains the terminology. 
Lemma 4.6 ([BOPT13b], Proposition 4.4). A periodic CS-fiber contains either a Cremer point or a Siegel point.

The drawback of using the lamination $\sim_{f}$ to model the dynamics of $f$ is that $\sim_{f}$ may incompletely reflect the properties of repelling periodic cutpoints of $J(f)$. In a lot of cases this does not happen. Indeed, if all $\sim_{f}$-classes are finite then by Theorem 4.4 there is a one-to-one correspondence between repelling and parabolic periodic cutpoints of $f$ and their preimages on the one hand and the (pre)periodic nondegenerate classes of $\sim_{f}$. However in the case when some $\sim_{f}$-classes are infinite this may no longer be the case.

E.g., suppose that a cubic polynomial $f$ has a fixed repelling point 0 at which $R_{f}(0)$ and $R_{f}\left(\frac{1}{2}\right)$ land, and no more repelling periodic cutpoints. Moreover, suppose that in each "half-plane" created by the cut $R_{f}(0) \cup\{0\} \cup R_{f}\left(\frac{1}{2}\right)$ there is a fixed Cremer point. Denote these fixed Cremer points by $a$ and $b$. Then a standard argument (see, e.g., [Mil00b]) shows that there are two quadratic-like Julia sets in $J(f)$, namely $J_{a}$ (containing $a$ ) and $J_{b}$ (containing $b$ ). Each of them corresponds to a quadratic Julia set with a Cremer fixed point, and by [BO06] the only monotone map of $J_{a}\left(J_{b}\right)$ onto a locally connected continuum is a collapse to one point. It follows that the only monotone map of $J$ onto a locally connected continuum is a collapse to one point. Hence the lamination $\sim_{f}$ identifies all points of the circle and misses the fact that $f$ has a fixed repelling cutpoint 0 .

Let $F$ be a fiber associated with an infinite $\sim_{f}$-class. We saw that $F$ may contain periodic repelling points cutting $F$ such that the corresponding leaves are not included in $\mathcal{L}_{\sim_{f}}$. By Proposition 40 [BCO11] there are at most finitely many repelling or parabolic cutpoints in $F$. To each such point $x$ we associate the convex hull of the set $\operatorname{Ar}_{f}(x)$. We add the edges of such convex hulls to $\mathcal{L}_{\sim_{f}}$. Then we add to $\mathcal{L}_{\sim_{f}}$ the edges of gaps corresponding to preimages of such points. Finally, we take the limit leaves of this family of leaves and add them to $\mathcal{L}_{\sim_{f}}$. This creates a new geo-lamination $\mathcal{L}_{f}$ called the geo-lamination generated by $f$. In this way we combine $\mathcal{L}_{\sim_{f}}$ with the rational lamination defined by Kiwi in Kiw04. In $\mathcal{L}_{f}$ we will distinguish between Fatou gaps corresponding to non-degenerate Fatou domains of $f$, infinite gap-classes $G$, and infinite gaps $H$ of $\sim_{f}$ such that $H \cap \mathbb{S}^{1}$ is one $\sim_{f}$-class subdivided by finitely many finite gaps or leaves and their preimages as in the definition of $\mathcal{L}_{f}$. 


\section{Proof of Theorem C}

Lemma 5.1 deals with the case when $\mathcal{L}_{f}$ has only degenerate leaves. We will assume that 0 is a fixed point of $f$.

Lemma 5.1. If all leaves of $\mathcal{L}_{f}$ are degenerate then $[f] \in \mathrm{CU}$, and 0 is the unique non-repelling periodic point of $f$. Moreover, if $\sim_{f}$ consists of one class, then 0 is a Cremer or Siegel fixed point.

Proof. We may assume that $\sim_{f}$ consists of one class coinciding with $\mathbb{S}^{1}$. By definition of $\mathcal{L}_{f}$ the map $f$ has no repelling periodic cutpoints, and by Theorem 4.4, the polynomial $f$ has no attracting or parabolic periodic points. By Lemma 4.6 the point 0 is a fixed Cremer or Siegel point. Suppose that there is a non-repelling periodic point $x \neq 0$ of $f$. Similar to the above $x$ is also a Cremer or a Siegel periodic point. Then by [Kiw00] there exists a repelling periodic point separating $x$ and 0 , a contradiction.

Lemma 5.2. If $[f] \in \mathrm{CU}$ then $\mathcal{L}_{\sim_{f}}=\mathcal{L}_{f}$.

Before we prove Lemma 5.2, we need to recall a description of quadratic invariant gaps given in [BOPT13a]. Let $G$ be a degree 2 invariant gap of some $\sigma_{3}$-invariant lamination. Then there is a unique edge $M$ of $G$ (the major of $G$ ) separating the circle into two arcs, one of which contains all vertices of $G$ and is of length at most $\frac{2}{3} ; M$ must be a critical leaf or a periodic leaf. Moreover, all edges of $G$ are iterated $\sigma_{3}$-preimages of $M$. Suppose that a quadratic invariant gap $G$ is a gap of $\sim_{f}$. By [BOPT13b, Theorem 7.7], if $M=\overline{\theta_{1} \theta_{2}}$ is a periodic major of $G$, then the external rays $R_{f}\left(\theta_{1}\right), R_{f}\left(\theta_{2}\right)$ land at the same point. This implies that if $\overline{\alpha \beta}$ is a (pre)periodic edge of $G$ then the external rays $R_{f}(\alpha), R_{f}(\beta)$ land at the same point.

Proof of Lemma 5.2. Suppose that $[f] \in \mathrm{CU}$ and $\mathcal{L}_{\sim_{f}} \neq \mathcal{L}_{f}$. Then $f$ has a periodic CS-fiber $F$. By Lemma 4.6 there exists a Cremer or Siegel periodic point $y \in F$. If $F$ is not invariant then $y$ is not fixed contradicting Definition 1.1. Thus $F$ is invariant, and we may assume that $y=0 \in F$ is a fixed Cremer or Siegel point. As above, by [BL02] the corresponding to $F$ invariant Fatou gap $G$ is of degree greater than 1. If $G$ is of degree 3 then $G=\mathbb{S}^{1}$. Since $[f] \in \mathrm{CU}$, the map $f$ does not have repelling periodic cutpoints. Since $G=\mathbb{S}^{1}$, then by Theorem 4.4 the map $f$ cannot have parabolic periodic points. Hence by definition in this case all leaves of $\mathcal{L}_{f}=\mathcal{L}_{\sim_{f}}$ are degenerate. Assume that $G$ is of degree 2. Since $[f] \in \mathrm{CU}$ has no repelling cutpoints, then $\mathcal{L}_{\sim_{f}} \neq \mathcal{L}_{f}$ implies that there is a parabolic periodic cutpoint $x$ of $F$. Since $f$ is cubic, by the Fatou-Shishikura inequality the union of the orbit of $x$ 
and the point 0 is the set of all non-repelling periodic points of $f$. In particular, there are no other periodic cutpoints of $F$.

Let $\widetilde{X}$ be the union of all rays landing at $x$ and $\{x\}$ itself. Some edges of the convex hull $X$ of $\operatorname{Ar}_{f}(x)$ are contained inside $G$ (otherwise $x$ would not be a cutpoint of $F$ ). Apply the map $\varphi_{G}$ which collapses to points all edges of $G$. It semiconjugates $\left.\sigma_{3}\right|_{G}$ to $\sigma_{2}$ so that the restriction of $\mathcal{L}_{f}$ onto $G$ induces a $\sigma_{2}$-invariant geo-lamination $\mathcal{L}_{f}^{2}$ which contains, by the above, some periodic leaves. By Proposition II.6.10b of [Thu85], the lamination $\mathcal{L}_{f}^{2}$ has an invariant gap $H$ of non-zero rotation number or the leaf $H=\overline{\frac{1}{3}} \frac{2}{3}$. Theorem II.5.3 of [Thu85] shows that if $H$ is a gap then either $H$ is a Siegel gap, or it is a gap with countably many vertices, or it is a finite gap. However in the first two cases it follows that the lamination $\mathcal{L}_{f}^{2}$ contains an isolated critical leaf. On the other hand, the construction of $\mathcal{L}_{f}^{2}$ implies that all non-degenerate leaves of $\mathcal{L}_{f}^{2}$ are either (pre)periodic with non-degenerate images, or limits of (pre)periodic, a contradiction. Thus, either $H=\overline{\frac{1}{3}} \frac{2}{3}$, or $H$ is a finite gap of rational rotation number.

Consider the convex hull $H_{1}$ of $\varphi_{G}^{-1}\left(H^{\prime}\right)$. Then $H_{1}$ has either the same number of vertices as $H$, or twice as many vertices as $H$ (if vertices of $H$ are $\varphi_{G}$-images of edges of $G$ ). We want to prove that there is an $f$-fixed point associated to $H_{1}$ such that external rays of $f$ whose arguments are vertices of $H_{1}$ land at that point. Indeed, suppose otherwise. Then by definition of our laminations we may assume that there are $\sigma_{2}$-pullbacks of $\varphi_{G}(X)$ accumulating on each edge of $H$. Let $\ell=\overline{a b}$ be an edge of $H$. Then the corresponding $\sigma_{3}$-pullbacks of $X$ will accumulate on the corresponding edge of $\overline{a_{1} b_{1}}$ of $H_{1}$. The corresponding cuts of $F$ formed by the corresponding pullbacks of $\widetilde{X}$ can be chosen so that their "vertices" (i.e., corresponding pullbacks of $x$ ) converge to a point $y_{\ell}$ belonging to the impression of $R_{f}\left(a_{1}\right)$ and the impression of $R_{f}\left(b_{1}\right)$. Thus, impressions of $R_{f}\left(a_{1}\right)$ and $R_{f}\left(b_{1}\right)$ are non-disjoint.

If $H_{1}$ and $H$ have the same number of vertices, it follows that the union $K$ of all impressions of angles with arguments which are vertices of $H_{1}$ is a continuum. If $H_{1}$ has twice as many vertices as $H$, for every vertex $l$ of $H$ there is an edge $\ell=\overline{u v}$ of $H_{1}$ such that $\varphi_{G}(\ell)=l$. By [BOPT13b, Theorem 7.7], the external rays $R_{f}(u), R_{f}(v)$ land at the same point. Hence in that case the union $K$ of impressions of angles which are vertices of $H_{1}$ is a continuum too. Clearly, $K$ is invariant and separated from impressions of rays with arguments which are not vertices of $H_{1}$ (either by the just discussed pullbacks of $\widetilde{X}$, or by the appropriate fibers approaching $F$ ). By [BCO11, Lemma 37] then $K$ is 
a fixed repelling or parabolic point. Since $[f] \in \mathrm{CU}, K$ is parabolic. Since $H$, and hence $H_{1}$, are of non-zero rotation number, the multiplier at $K$ is not one. On the other hand, $x$ is a Cremer or Siegel point. Thus, $f$ has at least two periodic points of multiplier not equal to 1 , a contradiction with $[f] \in \mathrm{CU}$. This shows that $\mathcal{L}_{\sim_{f}}=\mathcal{L}_{f}$.

Proof of Theorem $C$. In view of Lemma 5.2, it remains to prove that, for $[f] \in \mathrm{CU}$, the lamination $\mathcal{L}_{f}=\mathcal{L}_{\sim_{f}}$ is cubioidal. Let us prove that $\mathcal{L}_{f}$ has at most one rotational set $G$, and $G$ is invariant. Suppose that $G^{\prime}$ is a finite $\sim_{f}$-class. Then, by Theorem 4.4, it corresponds to a periodic repelling or parabolic cutpoint $y(G)=y$ of $J(f)$. Since $[f] \in \mathrm{CU}$, then, by Definition [1.1 (2), the point $y$ is parabolic and by Definition $1.1(3) y=0$. Hence $\mathcal{L}_{f}$ cannot have two finite rotational classes. Now, if $G$ is a Siegel gap of $\sim_{f}$ then there must exist a Siegel periodic point $y$ of $f$ inside $\varphi_{f}^{-1} \circ p_{\sim_{f}}(G)$; thus, $y=0$. Hence $\mathcal{L}_{f}$ has at most one rotational set $G$, and $G$ is invariant.

Again, let $G$ be a finite rotational $\sim_{f}$-class. Since $y(G)=y$ is a cutpoint of $J(f)$, by Definition $1.1(2)$, the point $y$ is parabolic. Hence there are parabolic domains attached to $y$. By Theorem 4.4 they are not collapsed by $\varphi_{f}$. Hence along at least one cycle of edges of $G$ such that the period of the endpoints of these edges is, say, $m$, there are Fatou gaps of period $m$ attached to $G$ and which do not correspond to one $\sim_{f}$-class as required in Definition 1.9, By [BOPT13a, Corollary $5.5]$ this implies that for every periodic leaf $\ell$ of $\mathcal{L}_{f}$ whose endpoints are of period $t$ there exists a Fatou gap of $\mathcal{L}_{f}$ of period $t$ attached to $\ell$. It remains to prove that such gaps cannot be contained in convex hulls of $\sim_{f}$-classes.

By the above the only hypothetical situation which we need to consider is as follows: there is a periodic finite gap or leaf $G$ of $\mathcal{L}_{f}$ with two cycles of edges on its boundary such that Fatou gaps which are not convex hulls of a single $\sim_{f}$-class are attached to one of these cycles of edges while Fatou gaps which are convex hulls of a single $\sim_{f}$-class are attached to the other cycle of edges. Denote by $H$ a Fatou gap which is one $\sim_{f}$-class attached to an edge of $G$; let $F$ be the corresponding CS-fiber. By Lemma 4.6 there is a Cremer or Siegel point $x \in F$. Since $[f] \in \mathrm{CU}$, the point $x=0$ is fixed and so $H$ is invariant. Clearly, the only way it can happen is when $G=\overline{0 \frac{1}{2}}$, a contradiction since if $\overline{0 \frac{1}{2}}$ is a leaf of $\mathcal{L}_{f}$ then from at least one side it has an attached Fatou gap which does not coincide with the convex hull of a $\sim_{f}$-class as desired (so that $\mathcal{L}_{f}$ is a CU-lamination).

We can partially reverse Theorem C. First we prove Lemma 5.3 . 
Lemma 5.3. If a cubic polynomial $f$ has no repelling cutpoints then it has a non-repelling fixed point.

Proof. Consider fixed external rays $R_{f}(0)$ and $R_{f}(1 / 2)$. If they land at the same point $w$ then by the assumptions $w$ is non-repelling as desired. Suppose that the ray $R_{f}(0)$ lands at $z$, the ray $R_{f}(1 / 2)$ lands at $y$, and $z \neq y$. By GM93] there exists either an invariant Fatou domain $U$ or a fixed point $x \in J(f) \backslash\{y, z\}$. In the first case $f$ has either an attracting or a Siegel fixed point, and we are done. In the second case there are two possibilities. First, a periodic ray $R$ may land at $x$. By the assumption about $R_{f}(0), R_{f}\left(\frac{1}{2}\right)$ the ray $R$ is not invariant, hence $x$ is a cutpoint. Since $f$ does not have repelling periodic cutpoints, $x$ is parabolic and we are done. Second, suppose that no periodic ray lands at $x$. Then $x$ is a Cremer fixed point, and we are done.

Lemma 5.4. Suppose that $\left(\sim_{f}, \mathcal{L}_{f}\right)$ is a cubioidal laminational pair, $f$ has no repelling periodic cutpoints and at most one periodic attracting point. Then $[f] \in \mathrm{CU}$.

Proof. By Lemma 5.3 we may assume that 0 is an $f$-fixed point, $\left|f^{\prime}(0)\right| \leqslant 1$, and if there is a fixed non-repelling point with multiplier not equal to 1 then $f^{\prime}(0) \neq 1$. By Definition $1.9, \mathcal{L}_{\sim_{f}}=\mathcal{L}_{f}$. By Definition 1.1 we need to show that all non-repelling periodic points of $f$ but perhaps 0 have multiplier 1. Assume the contrary: $f$ has a periodic non-repelling point $x \neq 0$, whose multiplier is different from 1 .

We need an observation concerning any parabolic point $y$ of $f$. By [Kiw02 either there is one cycle of rays landing at $y$, or there are two cycles of rays landing at $y$. In the first case inside each wedge at $y$ there is a parabolic Fatou domain attached to $y$. In the second case $a$ priori it may happen that there is one cycle of Fatou domains attached to $y$ inside one cycle of wedges at $y$, and the other cycle of wedges at $y$ contains no Fatou domains attached to $y$ inside them. However since $\left(\sim_{f}, \mathcal{L}_{f}\right)$ is cubioidal, it follows that if there are two cycles of rays (and hence wedges) at $y$, then there are two cycles of Fatou domains at $y$. Now we can consider several cases.

(1) Assume that 0 is attracting. Then there is an invariant Fatou domain $U$ containing 0. If $x$ is attracting, Cremer or Siegel then again by [Kiw00] there exists a repelling periodic cutpoint, a contradiction. Assume that $x$ is parabolic. Then the fact that the multiplier at $x$ is not 1 implies that $x$ cannot be a boundary point of $U$. By the above there are two cases. First, there may be one cycle of rays and one cycle of Fatou domains at $x$. Clearly, then we can find a point from the orbit of $x$ and a Fatou domain attached to it which can only be separated from 0 by a repelling periodic cutpoint, a contradiction. Second, there may 
be two cycles of Fatou domains at $x$. Together with $U$ they will form three cycles of Fatou domains of a cubic polynomial $f$, a contradiction.

(2) Assume that 0 is Cremer or Siegel. If $x$ is attracting, Cremer or Siegel then by Kiw00 there exists a repelling periodic cutpoint separating 0 and $x$ in $J(f)$, a contradiction. Suppose that $x$ is parabolic. As in (1), the fact that $f$ is cubic implies that there is exactly one cycle of Fatou domains at $x$. However this implies that there will be one of Fatou domains at one of the points of the orbit of $x$ which can only be separated from 0 by a repelling periodic cutpoint, a contradiction.

(3) Assume that 0 is parabolic. By the above there are two subcases here. First, assume that there are two cycles of Fatou domains at 0. Let $G$ be the convex hull of $\operatorname{Ar}_{f}(0)$. If $G$ is a gap, then each cycle of Fatou domains at 0 consists of at least two domains. If one of them is a cycle of attracting Fatou domains, then we have at least two attracting periodic points of $f$, a contradiction. If both are cycles of parabolic domains then clearly we cannot have a non-repelling periodic point $x \neq 0$. Thus, we may assume that $G$ is a leaf. Then having two cycles of Fatou domains at 0 (actually, each cycle in this case consists of just one Fatou domain) means having two cycles of Fatou gaps attached to $G$ which implies that $G=\overline{0 \frac{1}{2}}$. If both Fatou domains at 0 are parabolic, we cannot have a non-repelling periodic point $x \neq 0$. Hence one of the Fatou domains at 0 is attracting and the other one is parabolic. However in that case by our choice of $f$ we should have moved the attracting fixed point to 0 , a contradiction.

Second, assume that there is one cycle of Fatou domains and one cycle of rays landing at 0 . Then it is easy to see (similar to the arguments above) that there must exist a repelling cutpoint separating one of these Fatou domains at 0 from a specifically chosen Fatou domain at one of the points from the orbit of $x$. In any case, we get a contradiction with the assumption that $f$ has no repelling periodic cutpoints.

\section{Proof of the Second Part of Theorem B}

We need to prove that $\mathcal{L C} \cap \mathrm{CU}=\mathcal{L C} \cap \overline{\mathrm{PHD}}_{3}^{e}(\mathcal{L C}$ is the set of classes of polynomials with locally connected Julia sets). By the first part of Theorem B $\overline{\mathrm{PHD}}_{3}^{e} \subset \mathrm{CU}$. Hence we have to consider cubic polynomials $f$ such that $[f] \in \mathrm{CU} \backslash \overline{\mathrm{PHD}}_{3}^{e}$. By Theorem C, the laminational pair $\left(\sim_{f}, \mathcal{L}_{f}\right)$ is cubioidal. We may assume that $f \in \mathcal{F}_{n r}$.

6.1. Main analytic tools. According to [BOPT13b], there is a welldefined principal critical point $\omega_{1}(f)$ of $f$ that depends holomorphically on $f$ at least in a small neighborhood of $f$ in $\mathcal{F}_{n r}$. If $\lambda=f^{\prime}(0)$ is a root 
of unity, then $\omega_{1}$ is in a parabolic domain attached to 0 , in particular, the orbit of $\omega_{1}(f)$ converges to 0 .

Theorem 6.1 ([BOPT13b], Theorem B). If $f \in \mathcal{F}_{n r}$ and $[f] \notin \overline{\mathrm{PHD}}_{3}^{e}$ then there are Jordan domains $U^{*}$ and $V^{*}$ such that $f: U^{*} \rightarrow V^{*}$ is a quadratic-like map hybrid equivalent to $z^{2}+c$ with $c \in \overline{\mathrm{PHD}}_{2}$.

We will write $J^{*}$ for the Julia set of the quadratic-like map $f: U^{*} \rightarrow$ $V^{*}$, and $K^{*}$ for the filled Julia set of this map. Theorem 6.1 immediately implies that we may assume that $\mathcal{L}_{f}$ has some non-degenerate leaves. Lemma 6.2 allows us to not consider some polynomials.

Lemma 6.2 (Corollary 4.2 [BOPT13b]). Suppose that one of the following holds for $f$ :

(1) $f$ has a fixed parabolic point at which two cycles of parabolic Fatou domains are attached;

(2) $f$ has a locally connected Julia set and an invariant Siegel domain with two critical points on its boundary.

Then $[f] \in \overline{\mathrm{PHD}}_{3}$.

6.2. The description of cubioidal laminations [BOPT13a. A stand-alone quadratic invariant gap $U$ is a quadratic invariant gap $U$ of some lamination considered by itself (without the lamination). Quadratic means that $\sigma_{3}$ on $U^{\prime}=U \cap \mathbb{S}^{1}$ is two-to-one (except when $U$ has a critical edge $\bar{c}$ and the point-image of $\bar{c}$ has three preimages in $U^{\prime}$; critical means that the endpoints $\bar{c}$ map to one point). When studying such a gap $U$, an important role is played by the map $\psi_{U}: \operatorname{Bd}(U) \rightarrow \mathbb{S}^{1}$ collapsing all edges of $U$ and semi-conjugating the map $\left.\sigma_{3}\right|_{U}$ with $\sigma_{2}$.

The gap $U$ has a unique edge $M$ such that the arc $H(M)$ (called the major hole of $U=U_{M}$ ), which is the component of $\mathbb{S}^{1} \backslash M$ containing no points of $U^{\prime}$, is of length at least $\frac{1}{3}$ and at most $\frac{1}{2}$. Then $M$ is called the major (leaf) of $U$ and all other edges of $U=U_{M}$ map to $M$ in finitely many steps. The set $U^{\prime}$ consists of all points of $\mathbb{S}^{1}$ which never enter $H(M)$. In particular, $M$ never enters $H(M)$.

There are two types of majors of $U$. First, $U$ can be of regular critical type. Then $U$ has a critical major $M=\overline{\theta_{1} \theta_{2}}$. If a gap $U$ is of regular critical type then there exists a unique lamination such that $U$ is its gap. Basically, this lamination is obtained by taking pullbacks of $U$. This lamination is called the canonical lamination of the gap $U$.

Also, $U$ can be of periodic type with its major $M=\overline{\theta_{1} \theta_{2}}$ being a periodic edge of $U$ of period $k$. Call such $M$ a major (leaf) of periodic type. Clearly, $\left.\sigma_{3}\right|_{H(M)}$ wraps around the circle while for every $i, 1 \leqslant i \leqslant$ $k-1$ the map $\sigma_{3}$ is one-to-one on the circle arc with endpoints $\sigma^{\circ i}\left(\theta_{1}\right)$, 
$\sigma^{\circ i}\left(\theta_{2}\right)$ disjoint from $U$. Inside $H(M)$ there are points $\alpha, \beta$ such that $N=\overline{\alpha \beta}$ has the same image as $M$. We call $N$ a sibling leaf of $M$ and construct a gap $V_{M}$ with edges $M$ and $N$ consisting of all $x$ such that for every $n \geqslant 0$, the point $\sigma_{3}^{\circ n}(x)$ belongs to the closure of the arc with the same endpoints as $\sigma_{3}^{\circ n}(M)$ disjoint from $U$. Then $V_{M}$ is a quadratic gap of period $k$ (i.e., $\sigma_{3}^{\circ k}$ maps $V_{M}^{\prime}$ onto itself in a two-to-one fashion) called the vassal gap of $U$. If $M \neq \overline{0 \frac{1}{2}}$ then either $0 \in H(M)$ is the only fixed angle in $H(M)$ and the only angle which stays in $H(M)$ forever, or the same holds for $\frac{1}{2}$. By [BOPT13a] there is a unique lamination which has both $U$ and $V_{M}$ as its gaps. Similar to the regular critical case, this lamination is obtained by taking pullbacks of $U$ and $V_{M}$. It is called the canonical lamination of the gap $U$. Regardless of the type of the gap $U$, the corresponding canonical lamination is denoted by $\sim_{U}$ and the corresponding geo-lamination is denoted by $\mathcal{L}_{U}$.

Definition 6.3. A lamination $\mathcal{L}$ tunes a stand alone quadratic gap $U$ according to a quadratic lamination $\mathcal{L}_{2}$ if all edges of $U$ are leaves of $\mathcal{L}$, and the map $\psi_{U}$ transports the leaves of $\mathcal{L}$ in $U$ to the leaves of $\mathcal{L}_{2}$. If $\mathcal{L}$ and $\mathcal{L}_{1}$ are two laminations and $\mathcal{L} \supset \mathcal{L}_{1}$, say that $\mathcal{L}$ tunes $\mathcal{L}_{1}$.

Definition 6.4. A lamination $\mathcal{L}$ coexists with a stand-alone quadratic invariant gap $U$ if every leaf of $\mathcal{L}$ which intersects an edge $\ell$ of $U$ in $\mathbb{D}$ coincides with $\ell$. If the map $\psi_{U}$ transports the leaves of $\mathcal{L}$ in $U$ to leaves of a quadratic invariant lamination $\mathcal{L}_{2}$, we say that $\mathcal{L}$ weakly tunes $U$ according to $\mathcal{L}_{2}$. A lamination $\mathcal{L}$ coexists with a lamination $\mathcal{L}_{1}$ if no leaf of $\mathcal{L}$ intersects a leaf of $\mathcal{L}_{1}$ in $\mathbb{D}$ unless the two leaves coincide.

Theorem 6.5] is the main result of [BOPT13a].

Theorem 6.5. Let $\sim$ be a non-empty cubioidal lamination. Then (1) or (2) occurs (below $U$ is an invariant quadratic gap).

(1) The lamination $\sim$ tunes the canonical lamination $\sim_{U}$ according to a quadratic lamination $\asymp$ from the Main Cardioid, and if $U$ is of periodic type then the vassal gap $V(U)$ is a gap of $\sim$.

(2) The lamination $\sim$ coexists with the canonical lamination $\sim_{U}$ and weakly tunes $\sim_{U}$ on $U$ according to a quadratic lamination $\asymp$ from the Main Cardioid so that edges of $U$ are not leaves of $\sim$. Moreover, $U$ is of regular critical type.

6.3. The proof of the second part of Theorem B. Consider a cubic polynomial $f \in \mathcal{F}_{n r}$ with $[f] \in \mathrm{CU} \backslash \overline{\mathrm{PHD}}_{3}^{e}$. By Theorem C, the lamination $\mathcal{L}_{f}=\mathcal{L}_{\sim_{f}}$ is cubioidal. We may assume that all nonrepelling periodic points of $f$ but perhaps 0 have multiplier 1 .

Let us define a special set $Q_{f} \subset K^{*}$ for the polynomial $f$. If 0 is parabolic, by Lemma 6.2 there is a unique $f$-cycle $Q_{f} \subset K^{*}$ of Fatou 
domains at 0 . By Theorem 6.1, the map $\left.f\right|_{J^{*}}$ is hybrid equivalent to the appropriate quadratic polynomial $g$ with parabolic fixed point $a_{g}$ in its quadratic Julia set $J(g)$. Under this conjugacy $Q_{f}$ maps to the $g$-cycle $Q_{g}$ of Fatou domains at $a_{g}$. Otherwise 0 is a Siegel point (as Julia sets with Cremer points are not locally connected). Then the closure $Q_{f}$ of the Siegel domain at 0 is contained in $K^{*}$. Below, we consider the two cases of Theorem 6.5. We will assume that $U^{*}$ is sufficiently close to $K^{*}$ which can be arranged by passing to a suitable pullback.

Case (1) of Theorem 6.5. Denote by $U$ the quadratic invariant gap tuned by $\mathcal{L}_{f}$. Let $M$ be the major of $U$ and $t \in J(f)$ be the point corresponding to $M$. Then $t$ is a critical point (in the regular critical case) or a periodic cutpoint of $J(f)$ (in the periodic type case). As points of neither type belong to $J(g)$, we have $t \notin K^{*}$. Take the union $C$ of both external rays of $f$ landing at $t$. Let $T$ be the component of $\mathbb{C} \backslash C$ containing 0 . Since $K^{*}$ is positively distant from $C$, we may assume that $\overline{U^{*}} \subset T$. Choose $x \in J^{*}$ and all its backward orbits inside $J^{*}$. By the construction it is equivalent to considering all backward orbits of $x$ contained in $T$. The union $B$ of these backwards orbits is dense in $J^{*}$. On the other hand, by BOPT13a] the set of arguments of external rays landing at points of $B$ is dense in the basis $U^{\prime}$ of the gap $U$. These arguments will accumulate upon the arguments of external rays landing at $t$ which implies that their landing points converge to $t$, and hence that $t \in J^{*}$, a contradiction.

Case (2) of Theorem 6.5. In this case the invariant quadratic gap $U$ weakly tuned by $\mathcal{L}_{f}$ is of regular critical type, the major $M$ of $U$ does not belong to $\mathcal{L}_{f}$ and hence is contained in a critical gap $G$ of $\mathcal{L}_{f}$. Consider several cases depending on $G$.

Case A: the gap $G$ is a periodic infinite gap. Consider the quadratic lamination $\asymp$ from the Main Cardioid according to which $\mathcal{L}_{f}$ weakly tunes $U$. First, assume that $\asymp$ has an invariant Siegel gap $T$ with a critical edge corresponding to a critical leaf of $\mathcal{L}_{f}$ which is the second critical set of $\mathcal{L}_{f}$. Then $G$ corresponds to $T$ and is an invariant gap with a critical edge on which $\sigma_{3}$ is two-to-one. Hence $\mathcal{L}_{f}$ tunes $G$ and fits into case (1) of Theorem 6.5 considered above.

Second, assume that $\asymp$ corresponds to the lamination of a polynomial $g$ from the Main Cardioid which has a parabolic fixed point $a_{g}$. Then $\asymp$ has an invariant finite gap which must correspond to a finite invariant gap $H$ of $\mathcal{L}_{f}$. The (pre)periodic edges of $G$ contained in $U$ will be eventually mapped to edges of $H$; as $G$ is periodic, these leaves will also remain edges of $G$. Thus, $G$ "rotates" around $H$. 
Suppose that $G$ is quadratic and consider the major $M \subset G$ of $U$. The properties of quadratic maps imply that under the appropriate power of $\sigma_{3}$ the image of $M$ will be separated from $H$ by $M$ itself. This contradicts the properties of regular critical leaves according to which $M$ never enters $H(M)$. Hence $G$ is either cubic or is of degree 4 (in the latter case there must exist another critical Fatou gap in the orbit of $G$ ). However in both these cases it is easy to see that we have a contradiction with Theorem 6.1. Indeed, by Theorem 6.1 the fixed point 0 of $f$ corresponds to $a_{g}$, and hence the orbit of $G$ corresponds to the orbit of a Fatou domain of $g$ at $a_{g}$ which must be quadratic.

Case B: the gap $G$ is a preperiodic infinite gap. There is a Fatou domain $\widetilde{G}$ of $f$ corresponding to $G$. Note that $\widetilde{G}$ eventually maps to a periodic domain in $K^{*}$. Denote by $t \in \operatorname{Bd}(\widetilde{G})$ the cutpoint of $J(f)$ which separates $\widetilde{G}$ and $Q_{f}$. If $t \notin K^{*}$ then we obtain a contradiction as in the part of this proof corresponding to Case 1 of Theorem 6.5. Otherwise $t$ together with a small arc $I \subset \operatorname{Bd}(\widetilde{G})$ around it eventually maps to $Q_{f} \subset K^{*}$. By the definition of a polynomial-like Julia set $I \subset J^{*}$. On one side of $I$ (namely, inside $\widetilde{G}$ ) there are no points of $J(f)$, hence there are no points of $J^{*}$ on that side either. Well-known properties of locally connected quadratic Julia sets imply that then $I$ must be an arc from the boundary of a Fatou domain of $J^{*}$. However the entire $\widetilde{G}$ cannot be contained in $K^{*}$ because $K^{*}$ is quadratic-like, a contradiction.

Case $C$ : the gap $G$ is a preperiodic finite gap. Then $G$ corresponds to a critical point $t$ of $f$ whose image is still a cutpoint of $J(f)$. Clearly, $t \notin J^{*}$ because $J^{*}$ is quadratic-like. We may assume that $t \notin \overline{U^{*}}$. Then we obtain a contradiction, repeating the arguments from the part of this proof corresponding to Case 1 of Theorem 6.5.

\section{REFERENCES}

[Bea00] A.F. Beardon, "Iteration of rational functions: Complex Analytic Dynamical Systems", Springer 2000

[BCLOS10] A. Blokh, D. Childers, G. Levin, L. Oversteegen, D. Schleicher, An Extended Fatou-Shishikura inequality and wandering branch continua for polynomials, arXiv:1001.0953 (2010)

[BCO11] A. Blokh, C. Curry, L. Oversteegen, Locally connected models for Julia sets, Advances in Mathematics 226 (2011), 1621-1661

[BFMOT12] A. Blokh, R. Fokkink, J. Mayer, L. Oversteegen, E. Tymchatyn, Fixed point theorems for plane continua with applications, Memoirs of the American Mathematical Society, 224 (2013), no. 1053

[BL02] A. Blokh, G. Levin, Growing trees, laminations and the dynamics on the Julia set, Ergodic Theory and Dynamical Systems 22 (2002), 63-97 
[BO06] A. Blokh, L. Oversteegen, The Julia sets of quadratic Cremer polynomials, Topology and its Appl., 153 (2006), 3038-3050.

[BOPT13a] A. Blokh, L. Oversteegen, R. Ptacek, V. Timorin, Laminations from the Main Cubioid, preprint (2013)

[BOPT13b] A. Blokh, L. Oversteegen, R. Ptacek, V. Timorin, Quadratic-like dynamics of cubic polynomials, preprint (2013)

[BuHe01] X. Buff, C. Henriksen, Julia Sets in Parameter Spaces, Commun. Math. Phys. 220 (2001), 333 - 375

[DH8485] A. Douady and J. Hubbard, Étude dynamique des polynômes complex I \& II, Publ. Math. Orsay (1984-85)

[Fat20] P. Fatou, Sur les equations functionnelles, Bull. Soc. Mat. France 48 (1920)

[GM93] L.R. Goldberg, J. Milnor, Fixed points of polynomial maps. Part II. Fixed point portraits, Ann. scientifiques de l'É.N.S. $4^{e}$ sér., 26, No. 1 (1993), 51-98

[Hub93] J. H. Hubbard, Local connectivity of Julia sets and bifurcation loci: three theorems of Yoccoz, in: Topological Methods in Modern Mathematics, Publish or Perish (1993)

[Kiw00] J. Kiwi, Non-accessible critical points of Cremer polynomials", Ergodic Theory and Dynamical Systems, 20 (2000), Issue 05, 1391-1403

[Kiw02] J. Kiwi, Wandering orbit portraits, Trans. Amer. Math. Soc. 354 (2002), 1473-1485

[Kiw04] J. Kiwi, Real laminations and the topological dynamics of complex polynomials, Advances in Mathematics, 184 (2004), 207-267

[KvS06] O. Kozlovski, S. van Strien, Local connectivity and quasi-conformal rigidity of non-renormalizable polynomials, Proc. of the LMS, 99 (2009), 275-296

[MSS83] R. Mañé, P. Sad, D. Sullivan, On the dynamics of rational maps, Ann. Sci. École Norm. Sup. (4) 16 (1983), no. 2, 193-217.

[Mil00b] J. Milnor, Local connectivity of Julia sets: expository lectures, In: The Mandelbrot Set, Theme and Variations, Cambr. Univ. Press (2000), 67-116

[Mil06] J. Milnor, "Holomorphic dynamics", 3rd Ed., Matrix Press, 2006

[Shi87] M. Shishikura, On the quasiconformal surgery of rational functions, Ann. Sci. Ecole Norm. Sup., 20 (1987), 1-29

[Thu85] W. Thurston. The combinatorics of iterated rational maps (1985), in: "Complex dynamics: Families and Friends", ed. by D. Schleicher, A K Peters (2008), 1-108

(Alexander Blokh, Lex Oversteegen and Ross Ptacek) Department of Mathematics, University of Alabama at Birmingham, Birmingham, AL 35294

(Vladlen Timorin) Faculty of Mathematics, Laboratory of Algebraic Geometry and its Applications, Higher School of Economics, Vavilova St. 7, 112312 Moscow, Russia 
(Vladlen Timorin) Independent University of Moscow, Bolshoy Vlasyevskiy Pereulok 11, 119002 Moscow, Russia

E-mail address, Alexander Blokh: ablokh@math.uab.edu

E-mail address, Lex Oversteegen: overstee@math.uab.edu

E-mail address, Ross Ptacek: rptacek@uab.edu

E-mail address, Vladlen Timorin: vtimorin@hse.ru 